\title{
Design and Development of Marine Data Collecting Wave Rider - Towards a New Paradigm
}

\author{
KhaliqueEjaz Ahmed, B M Nagesh, KarthikSuriya Narayanan, Alan Joel \\ Cooper,Abhishek Kumar Singh \\ Department of Mechanical Engineering \\ Sir M Visvesvaraya Institute of Technology \\ Bengaluru, India \\ karthiksnarayanan28498@gmail. com
}

\begin{abstract}
As generations of human beings continue to strive to explore the universe, a separate universe has remained largely unexplored. The marine world encompasses $75 \%$ of the earth yet very little is known about it. Currently there are only two ways of collecting marine or oceanic data, one is by the use of buoys and the second is by the use large machines that have to be deployed by heavily manned teams into localized areas in the ocean. The data collected from these localized areas is then expanded for the entire ocean by the use of mathematical probabilistic models. Thus, this research project focuses on the design and development of an autonomous device that can collect and transmit relevant oceanic \& meteorological data to onshore data centers.
\end{abstract}

Keywords:marine, data, collection, wave, rider, ocean

\section{Introduction}

Oceanographic data are collected in two ways, they are, in situ methods and remote sensing methods. The most common method of remote sensing is by using satellites, but other methods are by using aircrafts, special buoys and even ships. Data obtained via satellite is a major asset for oceanographic research. In situ data sampling does not usually obtain sufficient spatial or temporal data resolution over the ocean that covers more than $70 \%$ of the planet. Satellite data when carefully calibrated and adjusted is blended with in situ data to obtain and provide the best assessment of global ocean conditions. This research project aims at creating an integrated device with the capability of recording $\&$ consolidating sub surface and in situ oceanic data points which are currently recorded by various different methods. The marine data collecting wave rider is an autonomous device that can simply be deployed into the ocean for the purposes of collecting oceanic data thereby reducing both the cost and labour currently required to collect oceanic data.[1]

All current methods of collecting sub surface and in situ data points come with inherent disadvantages such as high cost, high labour requirement and insufficient quality of data collected. All the above methods also make it difficult to integrate the data 
collected thereby making the analysis of the same also difficult. This device solves the issue by integrating the collection of all critical data points to one device which records the sub surface and in situ data points and transmits them to on shore data centres [2] in a consolidated manner. It reduces the labour requirement and cost of recording oceanic data while also providing a dependable source of high-quality data, which can be used in concurrence with satellite oceanography to analyse oceanic trends.

This will also enable the forecasting of weather conditions live to sailors thereby helping in their preparation to hazards and reduce their exposure to high risk weather conditions.[3][4] It can also help record oceanic data that can be used to monitor global warming, act as an early detection and warning system that warns against natural calamities. For more commercial purposes, the device can also be modified to perform the function of a water quality monitoring device that has applications in the pisciculture industry. [5] The key advantages of this device are that it significantly reduces the high risk and large amount of man power attributed to the collection of marine data. In its ideal form the device can be mass produced and deployed en masse, thereby acting as a net which continuously collects marine data while also mapping the ocean

\section{Critical data points identified}

- Sea Surface Temperature

- Sea Surface Salinity

- Ocean Currents

- Wind Speed and Direction

- Atmospheric Pressure

\section{$3 \quad$ List of Components}

- BME280 Pressure Sensor

- Electrically Isolated I2C Salinity Sensor

- YF-S201 Water Flow Sensor

- Anemometer

- DS18B20 Thermal Probe

- Raspberry Pi 4 Model B

\section{$4 \quad$ Circuit Design}

One of the key challenges in this project was the integration of all of the sensors and measuring devices utilized to record the oceanic data into one single cohesively operating circuit. Connecting the BME280 Sensor.The BME280 pressure sensor was connected by following the steps mentioned below: 
- Connect the VIN pin of the sensor to the $3 \mathrm{~V}$ pin in position 1 of the GPIO header of the Raspberry Pi. This enables power supply to the sensor. This is represented by the orange line in the circuit diagram.

- Connect the I2C pins to the corresponding I2C GPIO header pins at positions $3 \&$ 5 . These connections are represented by the orange (SDA) \& grey (SCL) lines in the circuit diagram.

- Finally, the GND pin of the Raspberry Pi is connected to the bus GND of the breadboard and the corresponding GND pin of the sensor is connected to the bus GND as well. This is represented by the black line in the circuit diagram.

\section{Connecting the DS18B20 Thermal Probe.} low:

The DS18B20 thermal probe was connected by following the steps mentioned be-

- Connect the DS18B20 to the breadboard and add a 4.7 kilo-ohm resistor between the VCC \& signal pins.

- Connect the power supply wire and GND of the sensor as specified previously.

- Finally, connect the signal wire to the Raspberry Pi through the GPIO pin at the 4th position. This enables communication between the Raspberry Pi and the sensor. This connection is represented by the pink line in the circuit diagram.

\section{Connecting the YF-S201 Flow Sensor}

The YF-S201 flow sensor was connected by following the steps mentioned below:

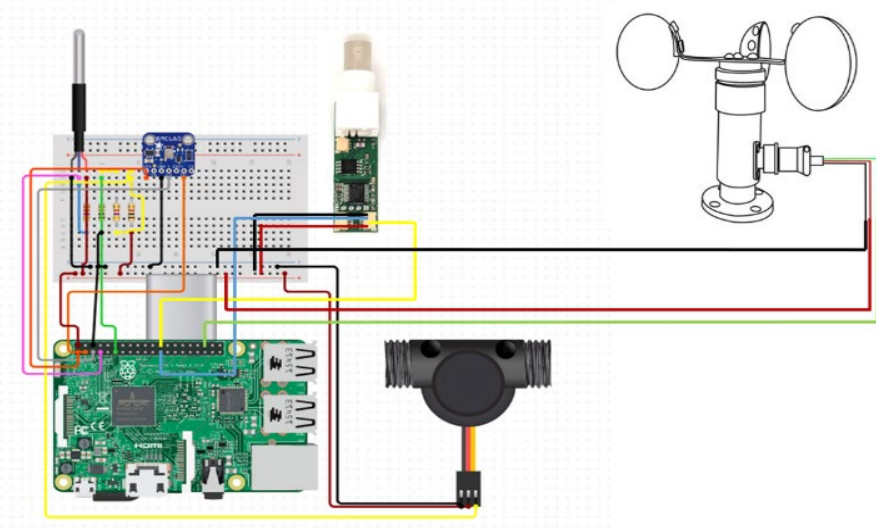

Fig.1.Circuit Diagram of Device Circuitry

- Connect the power supply wire and GND of the sensor as specified previously.

- Connect the signal wire of the sensor to the breadboard. 
- Next, connect the signal wire of the sensor to the Raspberry Pi via a 4.7 kilo-ohm resistor and a jumper wire which connects it to the GPIO pin the 6th position in the GPIO header. This entire circuit allows for communication between the sensor and the Raspberry Pi and the connections are represented by the yellow lines in the circuit diagram.

\section{Connecting the Salinity Sensor}

The salinity sensor was connected by following the steps mentioned below:

- Connect the power supply wire and GND of the sensor as specified previously.

- Now as this is a I2C sensor interface, similar to the BME280 sensor, it has another 2 pins that must be connected to the Raspberry $\mathrm{Pi}$, but there are no $\mathrm{I} 2 \mathrm{C}$ enabled pins left on the GPIO header. Thus, 2 additional I2C buses must be created $\&$ the connections must be made.

- This is done by connecting the pins by jumper wires, thus enabling communication between the sensor interface and the Raspberry Pi. The sensor interface is connected to the conductivity probe using a BNC connector, thereby completing the sensor setup. The connections are represented by blue (for SDA connection) and yellow (for SCL connection) lines in the circuit diagram.

\section{Connecting the Anemometer}

The anemometer was connected by following the steps mentioned below:

- Connect the power supply wire and GND of the sensor as specified previously.

- Finally, the signal wire from the anemometer is connected to the GPIO header of the Raspberry Pi using a jumper wire. The connection is made to the 36 th position pin and it is represented by a green line in the circuit diagram.

\section{$5 \quad$ Casing Design}

The casing design presented an especially difficult problem to solve, firstly as the device had to be maintained in an upright position for it to function properly and secondly it also had to float on the surface of water. These problems were solved with inspiration from the Lehigh Ocean Research Craft Autonomous, or LORCA design that has been developed by the Lehigh University Composite Lab [2]. This platform is designed to be robust enough for ocean deployment, while small enough to be launched by hand. The LORCA deck and hull are made as a single piece carbon fibre shell. The deck is domed so that the system is self-righting, and has only one small hatch for accessing internal components. The hull was designed to suite our needs for speed and cargo capacity. The casing designed for this project, as shown in the above figures, didn't have the complexities faced by the LORCA design as the wave rider is a relatively static device that simply floats along the ocean surface, while the LORCA was designed to act as an unmanned vehicle. Hence, incorporating the self-righting, 
highly domed design, as studied in the GáborDomokoset. al. paper [1], to the deck was the only requirement thereby simplifying the casing design. To ensure that the device would float it is as simple as utilizing Archimedes' principle. Archimedes' principle is represented by equation (1):

$$
\mathrm{Fb}=\rho \cdot \mathrm{g} . \mathrm{V}
$$

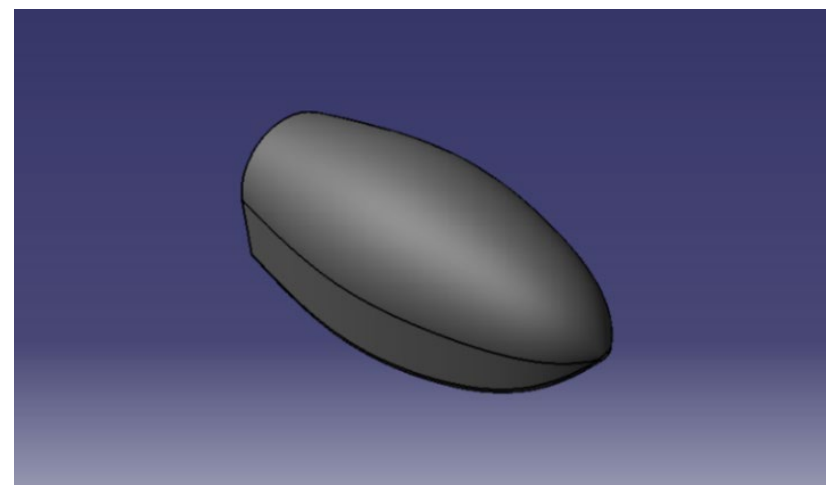

Fig.2.Isometric View of the Casing

As only the lower part of the device is expected to be submerged the volume in the equation above shall represent the submerged volume of the device. Considering the device as a hollow cuboid for simplicity of calculation the dimensions of the device are $0.6 \mathrm{X} 0.2 \times 0.24(1 \mathrm{X} \mathrm{b} \mathrm{X} \mathrm{h})$ in $\mathrm{m}$, with an oval shaped access hatch of the dimensions $0.14 \mathrm{X} 0.7(1 \mathrm{X} \mathrm{h})$ in $\mathrm{m}$, and the thickness of the casing is $0.02 \mathrm{~m}$.

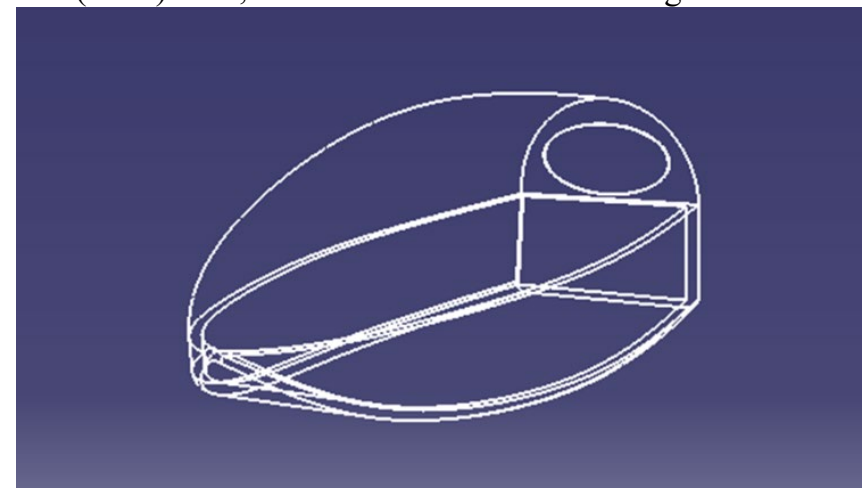

Fig.3.Wire Frame of the Casing showing the Access Hatch

Assuming that half of the device is submerged, the calculated submerged volume of the device is found to be $0.0144 \mathrm{~m} 3$. The density of oceanic water is about 1027 
$\mathrm{kg} / \mathrm{m} 3$. The added component weight can be ignored as it is far smaller when compared to the weight of the casing. Therefore, inputting the above values in (1), the value of force of buoyancy is obtained as $145.08 \mathrm{~N}$ and the weight of the displaced fluid as $14.7 \mathrm{~kg}$.

The density of the ABS material used to 3D print the casing is $1070 \mathrm{~kg} / \mathrm{m} 3$ and the effective volume off the device is $0.0064 \mathrm{~m} 3$. Thus, by using equation (2) shown below the mass of the device "m" can be obtained i.e.

$$
\mathrm{m}=\rho . \mathrm{V}
$$

Thus, the mass of the device is found to be $6.848 \mathrm{~kg}$ and therefore the force exerted by the device due to

gravity can be found by using the following equation (3):

$$
\mathrm{F}=\mathrm{m} \cdot \mathrm{g}
$$

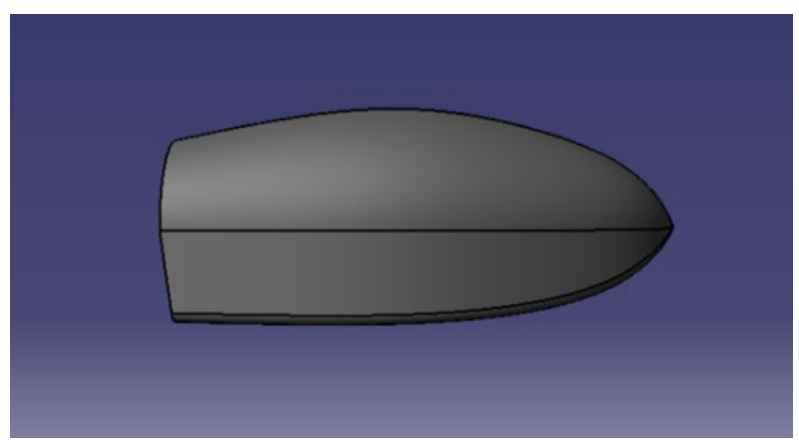

Fig.4.Side View of the Casing

Therefore, the force due to gravity is found to be 67.17 N. From the above calculations it is seen that the force of buoyancy is almost double that of the force of gravity and hence the device will float. Also due to the hollow nature of the device the hollow area within the device shall only aid its buoyancy characteristics.

\section{Conclusion \& Future Scope of Work}

This project was conceived to be a viable method of collecting oceanic data in a safer, easier and more comprehensive manner than what is currently prevalent in the world. With the integration of all pertinent data collecting devices that collect the prevalent critical data points from the oceans in a comprehensive manner this device achieves the goals it was set out for. The scope for the future of this device is also hugely expansive. With the implementation of non-conventional energy sources such as solar energy that allows it to recharge its battery the device can obtain a type of deploy and forget functionality that allows it to be out in the oceans for an indefinite period of 
time. By implementing swarm technology to a number of these devices it is possible to create a "net" of data collection that can monitor large areas of the ocean in real time and transmit that information back to on shore data centres for analysis. This particular feature holds great promise as it not only enables live data collection of oceanic data but along with the integration of Sonar technology into the device it could possibly help in the mapping of the oceanic surface topography. In a more consumer inclined application it can be used as water quality monitoring system in pisciculture farms and can also act as an early disaster detection system by monitoring weather conditions. Thus, the project endeavors to improve data collection rates from our oceans and there by hopefully making a small yet everlasting impact in the fight against global warming as well as reducing the risk that meteorological scientists face on their pursuit to improve the pre-existing knowledge of the undiscovered expanse of the ocean.

\section{References}

1. GáborDomokos and Péter L Várkonyi 2007 "Geometry and self-righting of turtles" Proc. R. Soc. B.27511-17

2. Larson, Scott, "Design and Construction of Unmanned Surface Vehicles" (2015). Theses and Dissertations. 2675.

3. K. D. Singh and S. T. Ahmed, "Systematic Linear Word String Recognition and Evaluation Technique," 2020 International Conference on Communication and Signal Processing (ICCSP), Chennai, India, 2020, pp. 0545-0548, doi: 10.1109/ICCSP48568.2020.9182044

4. S. T. Ahmed, H. K. Priyanka, S. Attar and A. Patted, "Cataract density ratio analysis under color image processing approach," 2017 International Conference on Intelligent Computing and Control Systems (ICICCS), Madurai, 2017, pp. 178-180, doi: 10.1109/ICCONS.2017.8250705

5. S. T. Ahmed, "A study on multi objective optimal clustering techniques for medical datasets," 2017 International Conference on Intelligent Computing and Control Systems (ICICCS), Madurai, 2017, pp. 174-177, doi: 10.1109/ICCONS.2017.8250704. 